Final report for project DE-FG02-03ER46050

\title{
Workshop on Fundamental Research Needs in Organic Electronic Materials
}

\author{
Held in Salt Lake City Utah, May 23-25 (2003)
}

PI: Prof. Zeev Valentine Vardeny

University of Utah

Salt Lake City, Utah 84112

\begin{abstract}
The workshop, Fundamental Research Needs in Organic Electronic Materials, jointly sponsored by DOE Basic Energy Sciences (BES) and EERE/BT was held on May 2325, 2003 at the University of Utah Campus in Salt Lake City, Utah for the purpose of identifying key scientific issues enabling the technological success of these materials. Approximately thirty key experts and world leaders in organic materials chemistry, transport physics, time-resolved and steady stated optical processes, organic spintronics, and device technology, gathered to stimulate new and revolutionary sciences.
\end{abstract}


The field of organic electronics has progressed enormously in recent years as a result of worldwide activity in numerous research groups. Advances have been made both in the fields of device science and fabrication as well as in the underlying Chemistry, Physics, and Materials Science. The impact of this field continues to influence many adjacent disciplines such as nanotechnology, sensors, and photonics. The advances in organic electronics have generated a vital and growing interest in organic materials research, and could potentially revolutionize future electronic applications. Recently, Senators Jeff Bingaman (D-NM) and Mike DeWine (R-OH) introduced legislation to authorize funding up to $\$ 50 \mathrm{M}$ per year of a Next Generation Lighting Initiative (NGLI), based on light emitting diodes (LED's) and organic light emitting diodes (OLED’s). The initiative, currently led by DOE Office of Energy Efficiency and Renewable Energy (EERE)/Building Technologies Program (BT), is expected to stimulate a major research and development effort in organic materials research for lighting and optoelectronic applications.

The growth of organic electronics has been impressive. The first commercial products were based on conducting polymer films, a business now with annual sales in the billiondollar range. OLED's and displays based on OLED's have been introduced to the scientific community more than a decade ago and to the market about half-dozen years; a large expansion in market penetration has been forecasted for the next decade. Efficiencies of small molecule and polymeric OLED's have reached figures close to their respective theoretical maxima. In addition, thin-film transistor based circuits, and electronic circuits incorporating several hundred devices on flexible substrates have been recently demonstrated. These levels of integration are sufficient for applications such as radio frequency identification tags. Organic transistors have also been successfully integrated with display elements and used as chemical sensors. Organic photodiodes have been fabricated with quantum efficiencies in excess of 50\%, and organic solar cells with power conversion efficiencies over $5 \%$ have been reported. Organic solar cell efficiencies are expected to increase with improvements in materials design and with the implementation of more sophisticated device architecture. There have been major strides made in understanding the physics of charge transport, luminescence, and charge transfer; as well in understanding interfaces between organic materials, and also between metal electrodes and organics. There continue to be advances made in the synthesis of new compounds and in improved synthetic procedures of important materials. Another area that has seen a tremendous growth is the use of novel low-cost fabrication procedures for a variety of inexpensive organic electronic devices. Finally a new field in organic electronics has recently emerged, namely organic spintronics, where the spin sense of injected carriers is preserved along the carrier drift distance; this field promises to be novel and exciting. Organic spin-valve devices have been already demonstrated at low temperature and the promise for a room temperature spin related device is high.

Despite this impressive set of advances, it is clear that more research needs to be done. $A$ workshop on "Fundamental Research Needs in Organic Electronic Materials", jointly sponsored by DOE Basic Energy Sciences (BES) and EERE/BT, was organized to identify key scientific issues enabling the technological success of this class of materials. Some specific technical topics discussed in this workshop included: (1) Charge Injection, 
(2) Electrical Transport, (3) Exciton Dynamics and Transport, (4) Organic Heterostructures, (5) Spin Dynamics, (6) Optical Processes, (7) Charge/ Energy Transfer, (8) Organic Film Structure, and (9) Theory (Quantum Chemistry / Condensed Matter Physics). This workshop brought together about 30 key experts and world leaders in organic materials chemistry, transport physics, time-resolved and steady stated optical processes, organic spintronics, and device technology, to stimulate new and revolutionary sciences. The workshop took place at the University of Utah in Salt Lake City, Utah, from Friday, May 23 to Sunday, May 25, 2003. Prof. Alan Heeger and the PI of this project, Prof. Valy Vardeny served as the Co-Chairmen of this meeting. There were 22 invited talks by experts from the USA and abroad, mainly from Europe and Japan with expertise in basic sciences and applications of organic semiconductors. The talks were given during the first two days of the meeting, while the third day was devoted to discussion and summary.

The following subsections are arranged according to the specific applications.

(a) General considerations; It was generally agreed that whereas organic devices can perform a wide variety of functions, the practical advantages principally stem from the following: (i) Large area electronics; this type of layout is unsuitable for the $\mathrm{Si}$ technology. Application areas where the speed (operating frequency) is not too high are good opportunities for organic electronics, especially where low cost is important. (ii) Low-cost electronics. Cheap throwaway electronics will become increasingly widespread. This area is another opportunity area where organics are well situated for practical implementations. (iii) Novel functionalities such as light emission and chemical and biological sensitivities that are not found in the more conventional Si (or amorphous silicon) electronics. (iv) White light emission. It is generally a difficult problem to generate white light using inorganic semiconductors. This acute problem can be naturally solved with OLED's since the optical gap of organic semiconductors span the entire visible spectral range. In addition, it is relatively easy to fabricate tandem OLED's, or OLED's from polymer blends that generate white light. (v) Organic Spintronics represents another opportunity for organic semiconductors. The long spin relaxation time in organic semiconductors resulting from the weak spin-orbit coupling in these low-Z materials, coupled with their flexibility enables them to self-adjust at interfaces with ferromagnetic spin injectors and makes organic semiconductors ideal candidates for novel spintronics applications.

(b) Organic light emitting diodes; The impressive increase in luminous efficiency of molecular and polymeric OLED's make them ideal candidates for solid-state lighting. External quantum efficiencies of $>10 \%$ (photons/electrons) and luminous efficiencies of $>200$ Lumens/Watt have been achieved with OLED's, making them one of the main candidates for low-cost and energy efficient lighting. Many issues still remain regarding the OLED lifetime at relatively high intensities that are needed for lighting. Another issue is the design, engineering and fabrication of OLED with white light emission (see section a-(iv) above). This will undoubtly be a major area of research activity in the coming years. 
Other research areas in need of attention include: (i) Carrier injection, related with ohmic and non-ohmic contacts. (ii) Spin effects, such as singlets versus triplets, and their relative formation cross-sections. (iii) Interfaces, such as organic/metal, organic/dielectric and organic/organic. (iv) Quenching of electroluminescence processes have been identified, such as quenching by metallic electrodes, by injected polarons and triplets, and quenching by electric field. (v) Theory and modeling. (vi) Morphology can drastically affect the OLED performance; a systematic study of morphology effects on OLED's performance is in order. (vii) Light extraction is an acute problem in OLED's. Various methods have been devised to extract more light, and their systematic study is needed. (viii) Material purity and new organic semiconductors; the exact relation between material purity and OLED performance is lacking. Also the question whether the field needs new organic active layers was discussed. (ix) Various ways of achieving white light were mentioned during the meeting and thoroughly discussed.

(c) Organic transistors; Organic field effect transistors, OFET's now routinely have mobilities $>0.1 \mathrm{~cm}^{2} /$ volt-sec, which is sufficient for many applications. This has been achieved for both p-channel and n-channel devices. The improved OFET devices have led to prototypes of electronic ID tags, electronic paper, and active matrix liquid crystal displays. There is also increasing interest in OFET-driven active matrix driven OLED/PLED displays. In universities and industrial laboratories in the U.S, Europe and Asia there has been remarkable progress in the application of soft-lithography, novel forms of patterning, and printing techniques to the fabrication of transistor based devices and circuits. Development of new materials and material families for both the semiconductor and insulator regions of the field effect devices are expected, needed and represent an important opportunity.

There are several issues in this area, which warrant detailed studies. These include the following: Grain boundary effects, injection into the FET device, the role of defects, and local crystallinity, reversed sweeping and cycling stress-effects, and long time-scale relaxation effects. Understanding the maximum room-temperature mobility remains an active issue: Is there a theoretical limit? More generally, detailed device modeling is needed with associated experimental work; it was stressed that details of the device operation are not the same as in the inorganic counterparts. Continued research on new materials, particularly n-channel FET materials and materials exhibiting ambipolar transport for logic and light-emission, will be needed.

(d) Photovoltaic (PV) and solar cells; This is an important focus area where a sizable amount of research is expected to be directed over the coming years. The most important potential advantage of organic PV is cost. The cost barrier to wider use of solar cells has been keenly felt by existing technologies (such as crystalline silicon and hydrogenated amorphous silicon) that have matured over the last two decades. Organic semiconductors, if they can achieve the reliability needed, may be able to provide a cost-effective alternative. At present, however power conversion efficiencies are in the neighborhood of $7 \%$ in the best organic solar cells. This will need to improve by at least a factor of two to $\sim 14 \%$ as a distant goal of the field. One of the biggest challenges is the carrier mobility that currently limits the charge collection efficiency. Improved mobilities will increase 
the organic solar cell fill factor and, thereby, will lead to higher solar cell efficiencies. Another challenge is the operating lifetime. Solar cells need to operate in intense sunlight without degradation for many years. It is not yet clear whether organic based systems can achieve this goal; the degradation mechanisms of organic solar cells need to be thoroughly explored. However the promise of cheap energy that is also good for the environment will propel future research in this direction.

The key issues identified in the physics and technology of organic PV and solar cells are the following; Charge generation, geminate recombination, electric and magnetic field effects on charge separation and recombination, carrier mobilities, polaronic effects, charge collection efficiency, organic semiconductors with optical gap in the near-ir spectral range to better match the solar energy spectrum, interpenetrating morphologies, exciton range and its dependence on the film morphology.

(e) Organic spintronics; The exploration of magnetic field control of organic electronic and optoelectronic devices has just begun, and appears to be a very promising research field for the near future. Applications include: spin-valves, memory devices, spintransistors, light emitting spin-valves, and giant magneto-resistance devices for sensors. So far research on organic spintronics has concentrated on organic magnets for potential application as spin injectors and organic spin-valve devices. In this conference there were encouraging announcements in both research avenues. It was announced that organic magnet based semiconductors such as $\mathrm{V}[\mathrm{TCNE}]_{\mathrm{x}}$ are stable at room temperature; apparently they were also studied as possible spin injection electrodes. Also organic spinvalves with $40 \%$ magneto-resistance were recently demonstrated at low temperature. This encouraging progress, coupled with the discovery of $100 \%$ spin injection capabilities of the half-metallic lanthanides indicate that organic spintronics represents a major opportunity; more research is needed with the required level of resources directed towards organic spintronics.

Key issues identified in this meeting were: magnetic field dependence of OPV and OLED performance, spin statistics in OLED's, spin diffusion range at room temperature in polymer and small molecule thin films, spin injection capabilities at room temperature, vertical versus horizontal devices, self-assembled monolayer devices.

The overall feeling among the participants was that organic electronics is a very fertile area of research with major opportunities that offer the potential for high impact. There is a large momentum already built by earlier work that should increase to meet these new challenges. The highly interdisciplinary nature of this field means that it will continue to be enriched by advances in other disciplines such as nanotechnology, optics, biopolymers, chemical and biological sensing, systems and circuits developments, new materials and novel methods of fabrication. 


\section{$\underline{\text { Publications }}$}

An article that summarized the conference was published in the Journal of Synthetic Metals;

"Executive Summary: Fundamental Research Needs in Organic Electronic Materials”, Z. V. Vardeny, A. J. Heeger, and a. Dodabalapur, Synth. Metals 148, 1 (2005). 